\title{
MiR-496 Inhibits the Proliferation Through Targeting LYN and AKT/mTOR Signaling Pathway in Gastric Cancer
}

\section{Rui Su}

Affiliated hospital of Chengde Medical college

Enhong Zhao ( $\nabla$ enhongzhao@163.com )

Affiliated hospital of Chengde Medical college

Jun Zhang

Capital Medical University Affiliated Beijing Friendship Hospital

\section{Research}

Keywords: AKT/mTOR signaling pathway, apoptosis, binding site, MiR-496, UTR

Posted Date: August 27th, 2020

DOI: https://doi.org/10.21203/rs.3.rs-61573/v1

License: (c) (i) This work is licensed under a Creative Commons Attribution 4.0 International License.

Read Full License 


\section{Abstract}

MiRNA operates as a tumor suppressor or carcinogen to regulate cell proliferation, metastasis, invasion, differentiation, apoptosis and metabolic process. In the present research, we investigated the effect and mechanism of miR496 in human gastric cancer cells. Cell proliferation was measured by CCK8 and clonogenic assay. Transwell test was performed to detect cell migration and invasion. Flow cytometry analysis was used to evaluate cell apoptosis. Bioinformatics software targetscan was used for the screening of miR-496's target gene. MiR-496 was down regulated in three gastric cancer cell lines, SGC790, AGS and MKN45 compared with normal gastric epithelial cell line GES-1. MiR-496 mimics inhibited the proliferation of AGS cells after the transfection for $48 \mathrm{~h}$ and $72 \mathrm{~h}$. The migration and invasion of AGS cells were also inhibited by the transfection of miR-496 mimics. In addition, miR-496 mimics induced the apoptosis through up regulating the levels of Bax and Active Caspase3 and down regulating the levels of $\mathrm{Bcl}-2$ and Total Caspase3. Bioinformatics analysis showed that there was a binding site between miR496 and LYN kinase (LYN). MiR-496 mimics could inhibit the expression of LYN in AGS cells. The overexpression of LYN blocked the inhibition of tumor cell growth, as well as the inhibition of AKT/mTOR signaling pathway induced by miR-496 in gastric cancer cells. In conclusion, miR-496 inhibited the proliferation through the AKT/mTOR signaling pathway via targeting LYN in gastric cancer cells. Our research provides a new potential target for clinical diagnosis and targeted treatment of gastric cancer.

\section{Background}

Gastric cancer is one of the most common human cancers, ranking the third in cancer-related deaths. Studies show that both genetic and epigenetic factors are involved in the pathogenesis of human tumors, including gastric cancer [1]. However, due to the low sensitivity and specificity of tumor markers, the detection of markers for gastric cancer is relatively limited [2]. Therefore, the discovery of new markers for the diagnosis of gastric cancer is an urgent problem. MicroRNAs (miRNAs) are non-coding RNAs with 21-25 bp [3]. They can reduce mRNA level by inhibiting mRNA translation or binding the 3'UTR region of mRNA, thus silencing their homologous target genes, playing an important role in cell processes and maintaining normal physiological environment [3]. Special miRNA operates as a tumor suppressor or carcinogen, so it is considered as a biomarker for early diagnosis and prognosis of cancer [4-6]. These miRNAs also regulate cell proliferation, metastasis, invasion, differentiation, apoptosis and metabolic process [4-6].

MiR-496 is located on human chromosome 14 and has unknown biological functions [7]. Limited researches have shown that low expression of miR-496 is related to aging process $[8,9]$, and plays an important role in the formation and differentiation of bone cells [10]. MiR-496 overexpression inhibits breast cancer cell proliferation through MBD2-dependence[11]. At present, there are few reports of miR496, biological function of which in tumorigenesis is still unclear. In addition, miR-496 has not been reported in the proliferation and metastasis of gastric cancer cells. Therefore, we investigated the role and mechanism of miR-496 in vitro to provide new approach for clinical diagnosis and treatment of gastric cancer. 


\section{Material And Methods}

\subsection{Cell culture and transfection}

Human normal gastric epithelial cell line GES-1 and gastric cancer cell lines BGC-823, SGC-790, AGS, MKN45 and MKN28 were all purchased from Cell Bank of The Chinese Academy of Sciences (Shanghai, China). DMEM with $10 \%$ FBS was used for cell culture. MiR-496 mimics, miRNA mimics negative control and LYN kinase (LYN) overexpression plasmid were purchased from GenePharme (Shanghai, China) and transfected into AGS cells using lipofectamine2000 (Invitrogen, CA, USA) according to manufacturer's protocol.

\subsection{Fluorescence quantitative PCR (qPCR)}

After the transfection for $24 \mathrm{~h}$, total RNA was extracted from AGS cells by TRIzol reagent (Invitrogen, USA) and reverse transcript to $\mathrm{CDNA}$ using M-MLV Reverse transcriptase and TaqMan ${ }^{\mathrm{TM}}$ MicroRNA Reverse Transcription Kit (Invitrogen, USA). QPCR was performed to detect the level of miR-496 and LYN using the qPCR kit according to manufacturer's protocol (Invitrogen, USA). The relative expression of miR-496 and LYN was analyzed using $2^{-\Delta \Delta C t}$ method.

\subsection{Western blot}

After the transfection for $48 \mathrm{~h}$, whole-cell protein was extracted using a RIPA lysis buffer. The protein concentrations were detected by bicinchoninic acid (BCA) method. Then, protein extracts were separated using SDS-PAGE and transferred to a polyvinylidene fluoride (PVDF) membrane. After blocking with skimmed milk, the protein was incubated with the primary antibodies for $1 \mathrm{~h}$, followed by secondary antibodies for $1 \mathrm{~h}$. primary antibodies were all purchased from Cell Signaling Technology (Danvers, MA, USA), including anti-Bcl-2, anti-Bax, anti-total Caspase-3, anti-Cleaved Caspase-3, anti-GAPDH, antivimentin, anti-Cyclin D1, anti-Snail2, anti-p-AKT (Ser473), anti-p-mTOR(Ser2448), and anti-p70S6K (1:1000).

\subsection{CCK8 assay}

The proliferation of AGS cells was detected by Cell Counting Kit-8 (CCK8). After transfection, cells were transfferd into a 96 -well plate (1000 cells/well). CCK-8 reagent was added into cells at a series of time points after the transfection ( $0 \mathrm{~h}, 24 \mathrm{~h}, 48 \mathrm{~h}$ and $72 \mathrm{~h}$ ). Cell viability was represented by OD value at 450 $\mathrm{nm}$.

\subsection{Clonogenic assay}

After the transfection, about 500 cells were transferred into a $6 \mathrm{~cm}$ dish and cultured at $37{ }^{\circ} \mathrm{C}$ for 2 weeks. Then, the colonies were fixed with methanol for $20 \mathrm{~min}$ and stained by $0.1 \%$ crystal violet for 30 min. Visible colonies were counted separately by two researchers. 


\subsection{Transwell}

For cell migration, AGS cells were transferred into the up chamber of transwell inserts after transfection

( $2 \times 10^{5}$ cells). Serum-free medium was added into the lower chamber of transwell inserts. After incubation for $48 \mathrm{~h}$, AGS cells were fixed with methanol for $10 \mathrm{~min}$ and stained with $0.1 \%$ crystal violet for 10 min. The migrated cells were counted in 5 random fields. For cell invasion, transwell inserts were pre-coated with Matrigel.

\subsection{Flow cytometry analysis}

After transfection for $48 \mathrm{~h}$, the apoptosis of AGS cells was detected using Annexin-V and PI (BD Biosciences, Franklin Lakes, NJ, USA) following the manufacturer's protocol. The percentage of apoptotic cells was analyzed on a FACScalibur flow cytometer using CellQuestPro software (Becton Dickinson, USA).

\subsection{Statistical analysis}

A one-way analysis followed by post hoc Bonferroni test was performed to analyze the differences between groups. All data in the present study were analyzed and plot with GraphPad Prism 8 (GraphPad Software Inc., San Diego, California, USA). All experiments were performed in triplicate. $P<0.05$ was considered statistically significant.

\section{Results}

\subsection{MiR-496 inhibited the proliferation in gastric cancer cells.}

We first detected the expression of miR-496 in gastric cancer cell lines (BGC-823, SGC-790, AGS, MKN45 and MKN28) by qPCR with the normal gastric epithelial cell line GES-1 as a control. MiR-496 was down regulated in SGC-790, AGS and MKN45 compared with GES-1 cells $(P<0.05$, Fig. $1 \mathrm{~A})$. Then, the miR-496 mimics or miRNA mimics negative control was transfected into AGS cells to generate miR-496 overexpressed (miR-496) or negative control (NC) cells, respectively. The level of miR-496 increased significantly in the miR-496 group compared with the NC group $(P<0.05$, Fig. 1B). The proliferation was detected using CCK8 and clonogenic assay. As shown in Fig. $1 \mathrm{C}$, the $\mathrm{OD}_{450}$ declined significantly after the transfection of miR-496 mimics for $48 \mathrm{~h}$ and $72 \mathrm{~h}$ compared with $\mathrm{NC}$ cells $(P<0.05)$. The colony number also markedly declined after transfection and incubated for 2 weeks compared with the NC cells $(P<0.05$, Fig. 1D). These data indicated that miR-496 could inhibit the proliferation of human gastric cancer cells.

\subsection{MiR-496 inhibited the migration and invasion in gastric cancer cells.}

Next, the migration and invasion of AGS cells with the transfection of miR-496 mimics were detected using transwell assay. As shown in Fig. 1E, the migration cell number decreased after the transfection of miR-496 mimics $(P<0.05)$; the invasion cell number also declined after the transfection of miR-496 
mimics compared with the miRNA mimics negative control $(P<0.05)$. These results suggested that miR496 couls inhibit the metastasis in gastric cancer cells

\subsection{MiR-496 promoted the apoptosis in gastric cancer cells.}

Then, we detected the apoptosis of AGS cells using flow cytometry. As shown in Fig. 2A, the percentage of apoptosis cells in AGS cells transfected with miR-496 mimics $(9.50 \pm 0.75 \%)$ increased compared with the NC cells $(4.03 \pm 0.50 \%)(P<0.05)$. In order to analyze the molecular mechanism by which miR-496 promotes tumor cell apoptosis, western blot was used to detect the expression of critical apoptotic factors in each group, including Bax, Active Caspase3, Total Caspase3 and Bcl-2. As shown in Fig. 2B-F, miR-496 mimics unregulated the expression of Bax and Active Caspase3, but downregulated the expression of Total Caspase 3 and $\mathrm{Bcl}-2(P<0.05)$. These results proved that miR-496 promoted the apoptosis through regulating apoptotic factors in gastric cancer cells.

\subsection{MiR-496 inhibited the expression of LYN in gastric cancer cells.}

Next, we predicted the potential binding targets of miR-496 using the online analysis tool targetscan (http://www.targetscan.org/vert_71/) [12]. According to the analysis on targetscan, there was a binding site between miR-496 and 3 'UTR of LYN (Fig. 3A). After transfected with miR-496 mimics, the mRNA and protein level of LYN significantly declined compared with the NC cells according to the results of qPCR (Fig. 3B) and western blot (Fig. 3C). Thus, we hypothesized that LYN was the downstream direct target of miR-496 in gastric cancer cells.

\subsection{LYN blocked the inhibition of tumor cell growth induced by miR-496 in gastric cancer cells.}

In order to elucidate whether miR-496 exerts tumor suppressive effect in gastric cancer through LYN, we transfected miR-496 mimics and LYN overexpression plasmid (miR-496\&LYN) in AGS cell line at the same time (Fig. 4A), and detected the cell proliferation and apoptosis with miR-496 mimics as a control. As shown in Fig. 4B, $\mathrm{OD}_{450}$ of miR-496\&LYN group increased markedly compared with the miR-496 group $(P<0.05)$. The percentage of apoptosis cells in miR-496\&LYN group $(2.20 \pm 0.36 \%)$ declined compared with the miR-496 group ( $8.31 \pm 0.35 \%$ ) (Fig. 4C, $P<0.05)$. These data indicated that miR-496 might inhibit the growth of gastric cancer cells through suppressed the expression of LYN.

\subsection{MiR-496 inhibited the AKT/mTOR signaling pathway via LYN in gastric cancer cells.}

Finally, we explored the molecular mechanism by which miR-496/LYN regulated the growth of gastric cancer. AKT/mTOR signaling pathway widely involves in the origin and development of solid tumors and plays a key role in the proliferation and apoptosis of tumor cells $[13,14]$. In our previous studies, we found that the AKT/mTOR pathway was down-regulated by LYN knockdown in AGS cells, including decreased levels of p-AKT, p-mTOR and down-stream effector p70. The AKT pathway activator IGF-1 was found to reverse the inhibitory effects of LYN knockdown on the proliferation, migration, and invasion in AGS cells. 
Thus, in this research, we detected the expression of protein related to AKT/mTOR signaling pathway in each group using western blot (Fig. 5A). The phosphorylation levels of AKT (Fig. 5B) and mTOR (Fig. 5C)

and the levels of Cyclin D1 (Fig. 5D) and P70 (Fig. 5E) were significantly inhibited by the transfection of miR-496 mimics and rescued by the transfection of LYN overexpression plasmid. LYN overexpression blocked the inhibition of AKT/mTOR signaling pathway induced by miR-496 mimics. Out results indicated that miR-496/LYN inhibited the tumor growth through suppressing the AKT/mTOR signaling pathway.

\section{Discussion}

In recent years, the role of miR-496 in human tumors has been gradually revealed. LncRNA DANCR was found to involve in the progression of lung cancer through sponging miR-496 and then regulate the level of mTOR [7]. MiR-496 also can suppress gene expression and the proliferation in breast cancer cell line MCF-10A, MCF-7 and MDA-MB-231 [11]. In another report, miR496 is down regulated by Human papillomavirus (HPV), notably type 16 and inhibit the post-transcriptional control of the transcription factor E2F2 in oropharyngeal cancer [15]. In the present study, we found that miR-496 could inhibit the proliferation, migration and invasion, and induce the apoptosis in gastric cancer cells. Our data proved that miR-496 involved in the progression of human gastric cancer.

Studies have shown that miRNA, which is combined with RNA-induced silencing complex (RISC), can recognize and bind $3^{\prime} U T R$ of target genes by incomplete or complete matching, and achieve post transcriptional regulation of target gene expression level $[4,16,17]$. In mammals, miRNA usually binds to the 3'UTR of target gene through incomplete pairing, which affects the translation of target mRNA [17, 18]. In addition, a miRNA can regulate multiple mRNA, and different miRNAs also can coordinate to regulate one mRNA molecule [17]. It is predicted that about $1 / 3$ of the protein coding genes in human cells are regulated by miRNA [19]. MiRNAs and their target molecules form a complex regulatory network to control control cell activity [19]. In the present research, we analyzed the potential target of miR-496 on targetscan. A binding site was found between miR-496 and the 3'UTR of LYN. QPCR result showed that miR-496 mimics significantly inhibited the expression of LYN in AGS cells, suggesting that LYN was a downstream target of miR-496 in gastric cancer. Then, we detected the proliferation and apoptosis in both miR-496 and LYN overexpressed cells with the only miR-496 overexpressed cells as a control. The results proved that LYN blocked the increase of cell proliferation and decrease of apoptosis induced by mir-496 in AGS cells. LYN is a member of the Src family tyrosine kinases (SFKs) and operates as a prooncogene in the progression of human tumor $[20,21]$. Our previous studies showed that knockdown of LYN inhibited both proliferation and metastasis and resulted in the activation of the mitochondrial apoptotic pathway in AGS cells.

The progression of gastric cancer involves many signaling pathways [22-25]. Our previous study found that LYN can promote cell proliferation and metastasis in AGS cells by activating AKT/mTOR signaling pathway. AKT/mTOR can regulate and stimulate cell growth by aggregating and integrating stimulating signals from nutrition, growth factors, energy, and environmental stresses to the cell, and is the hub of 
many important signal transduction pathways in the cell, involving in various biological functions such as gene transcription, protein translation, ribosome synthesis and apoptosis [26]. Studies in recent years show that AKT/mTOR signaling pathway is closely related to the occurrence, development and treatment of human tumors [27]. Therefore, we further studied the effect of miR-496/LYN on AKT/mTOR signaling pathway. MiR-496 could significantly inhibit the phosphorylation of AKT and mTOR, as well as the expression of P70 and Cycling D1, which were blocked by LYN overexpression. Therefore, we hypothesizes that miR-496/LYN/AKT/P70 may be a cell growth regulatory pathway in gastric cancer cells. However, the role of miR-496 and LYN in gastric cancer still remains unclear and needs further exploration.

In conclusion, miR-496 inhibits the proliferation, metastasis and induces the apoptosis through targeting LYN and inhibiting the AKT/mTOR signaling pathway in gastric cancer. Our research provides a new potential target for clinical diagnosis and targeted treatment of gastric cancer.

\section{Abbreviations List}

LYN: LYN kinase

BCA: bicinchoninic acid

PVDF: polyvinylidene fluoride

HPV: Human papillomavirus

RISC: RNA-induced silencing complex

\section{Declarations}

\section{Ethics approval and consent to participate}

This research was approved by the ethics committee of the Affiliated Hospital of Chengde Medical College.

\section{Consent for publication}

Not applicable

\section{Availability of data and material}

The data that support the findings of this study are available from the corresponding author upon reasonable request.

Competing interests 
The authors declare no conflict of interest.

\section{Funding}

The present study was supported by the Beijing Municipal Science \& Technology Commission (No.D171100006517003).

\section{Authors' contributions}

RS and EZ mainly performed the experiments and analyzed data. JZ helped with the experiments and analyzed the data. RS was a major contributor in writing the manuscript. All authors read and approved the final manuscript.

\section{Acknowledgements}

Not applicable

\section{References}

1. Piazuelo, M.B. and P. Correa, Gastric cancer: Overview. Gastroenterology Clinics of North America, 2013. 44(3): p. 192-201.

2. Gotoda, T., H. Yamamoto, and R.M. Soetikno, Endoscopic submucosal dissection of early gastric cancer. Journal of Gastroenterology. 41(10): p. 929-942.

3. Xue, M., Y. Zhuo, and B. Shan, MicroRNAs, Long Noncoding RNAs, and Their Functions in Human Disease. Methods Mol Biol, 2017: p. 1-25.

4. Yan, C., et al., MiR-760 suppresses non-small cell lung cancer proliferation and metastasis by targeting ROS1. Environmental Science \& Pollution Research International, 2018. 25(19): p. 1838518391.

5. Mario, A. and C.M. Croce, Downregulation of miR-15a and miR-16-1 at $13 q 14$ in Chronic Lymphocytic Leukemia. Clinical Chemistry, 2020(4): p. 4.

6. Gu, C., et al., Upregulation of miR-324-5p Inhibits Proliferation and Invasion of Colorectal Cancer Cells by Targeting ELAVL 1. Oncology Research Featuring Preclinical \& Clinical Cancer Therapeutics, 2019.

7. Lu, Q.C., et al., LnCRNA-DANCR contributes to lung adenocarcinoma progression by sponging miR496 to modulate mTOR expression. Journal of Cellular and Molecular Medicine.

8. Rubie, C., et al., microRNA-496 - A new, potentially aging-relevant regulator of mTOR. Cell Cycle. 15(8): p. 1108-1116.

9. Hooten, N.N., et al., microRNA Expression Patterns Reveal Differential Expression of Target Genes with Age. Plos One, 2010. 5.

10. Huang, J. and L. Chen, $I L-1 \beta$ Inhibits Osteogenesis of Human Bone Marrow-Derived Mesenchymal Stem Cells by Activating FoxD3/microRNA-496 to Repress Wnt Signaling. 2017. 55(7). 
11. Alvarado, S., et al., Methylated DNA Binding Domain Protein 2 (MBD2) Coordinately Silences Gene Expression through Activation of the MicroRNA hsa-mir-496 Promoter in Breast Cancer Cell Line. Plos One, 2013. 8.

12. Vikram, A., et al., Predicting effective microRNA target sites in mammalian mRNAs. eLife. 4.

13. Tapia, O., et al., The PI3K/AKT/mTOR pathway is activated in gastric cancer with potential prognostic and predictive significance. Archiv Für Pathologische Anatomie Und Physiologie Und Für Klinische Medicin. 465(1): p. 25-33.

14. Owusu-Brackett, N., M. Shariati, and F. Meric-Bernstam, Role of PI3K/AKT/mTOR in Cancer Signaling: Applications in Precision Medicine. 2019.

15. Dayna, M., et al., Human papillomavirus 16 E6 modulates the expression of miR-496 in oropharyngeal cancer. Virology. 521: p. 149-157.

16. Chang, L., et al., miR-145 targets the SOX11 3'UTR to suppress endometrial cancer growth. 2017. 7(11): p. 2305-2317.

17. Cambronne, X.A., et al., Capturing microRNA targets using an RNA-induced silencing complex (RISC)trap approach. Proceedings of the National Academy of Sciences of the United States of America. 109(50): p. 20473-20478.

18. Vishnoi, A. and S. Rani, MiRNA Biogenesis and Regulation of Diseases: An Overview. 2017: Springer New York.

19. Lewis, B.P., C.B. Burge, and D.P. Bartel, Conserved Seed Pairing, Often Flanked by Adenosines, Indicates that Thousands of Human Genes are MicroRNA Targets.120(1): p. 0-20.

20. Takata, M., et al., Tyrosine kinases Lyn and Syk regulate B cell receptor-coupled Ca2+ mobilization through distinct pathways. Embo Journal. 13(6): p. 1341-1349.

21. Croucher, D., et al., A novel signaling pathway in basal breast cancer involving Lyn and the atypical kinase SgK269/PEAK1. 2013. 73(10): p. 1435-1437.

22. Zhang, H.Y., et al., LC-0882 targets PAK4 and inhibits PAK4-related signaling pathways to suppress the proliferation and invasion of gastric cancer cells. 2017. 9(6): p. 2736.

23. Wei, G.H. and X. Wang, IncRNA MEG3 inhibit proliferation and metastasis of gastric cancer via p53 signaling pathway. 2017. 21(17): p. 3850-3856.

24. Ma, D.H., et al., miR-93-5p/IFNAR1 axis promotes gastric cancer metastasis through activating the STAT3 signaling pathway. 2017. 408: p. 23-32.

25. Pan, T., et al., miR-944 inhibits metastasis of gastric cancer by preventing the epithelialmesenchymal transition via MACC1/Met/AKT signaling. Febs Open Bio, 2017. 7(7).

26. Lopiccolo, J., et al., Targeting the PI3K/Akt/mTOR pathway: Effective combinations and clinical considerations.11(1-2): p. 0-50.

27. Lim, H.J., P. Crowe, and J.-L. Yang, Current clinical regulation of PI3K/PTEN/Akt/mTOR signalling in treatment of human cancer. Journal of Cancer Research \& Clinical Oncology. 141(4): p. 671-689. 
Figures

A

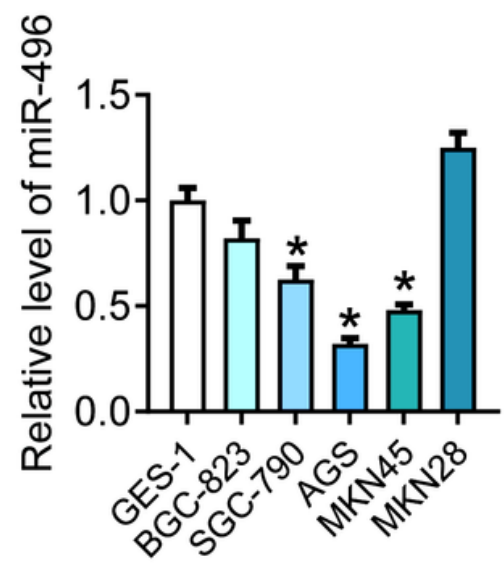

B

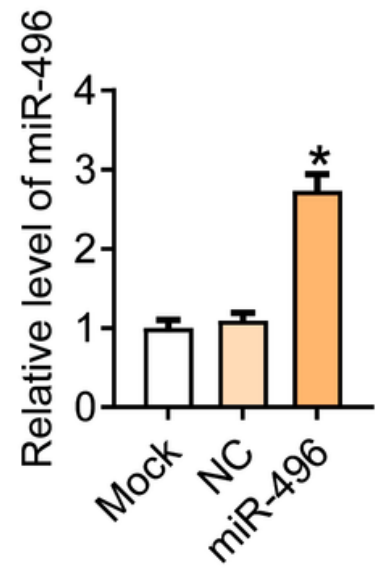

C The proliferation of AGS cells

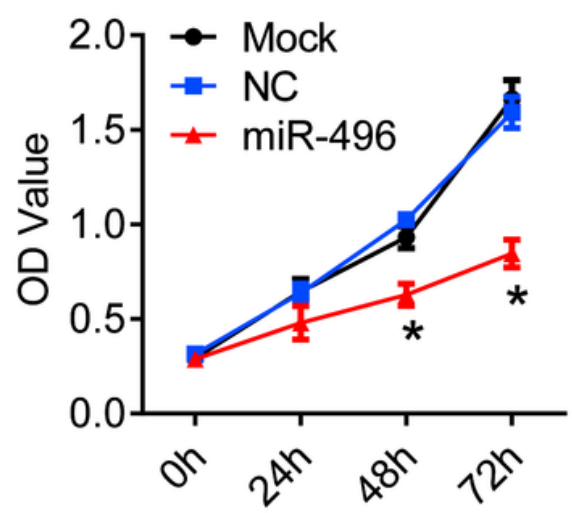

$\mathrm{D}$
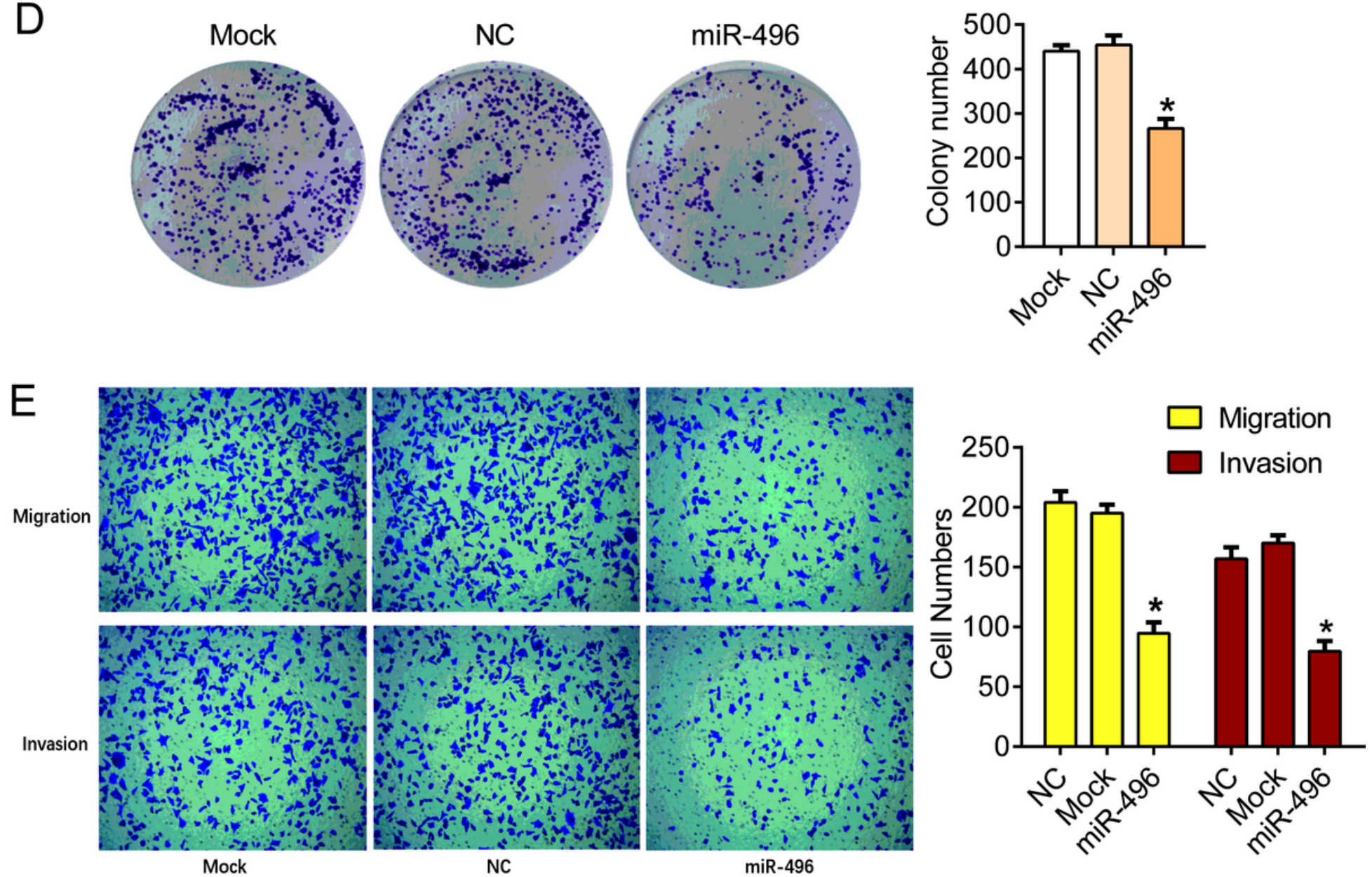

Figure 1

MiR-496 inhibited the proliferation and metastasis in gastric cancer cells. A. QPCR was performed to detect the level of miR-496 in AGS cells. B. the level of miR-496 in AGS cells transfected with miR-496 mimics was detected by qPCR. Relative level of miR-496 was analyzed using 2- $\Delta \Delta C$ t method and normalized to mock group. C. The proliferation of AGS cells was determined using CCK8 assay. OD value $(450 \mathrm{~nm})$ was measured every 24 h. D. Clonogenic assay was used to detect the proliferation of AGS cells. 
Colony number was counted after the cultural for 2 weeks. E. the migration and invasion of AGS cells was detected by transwell assay after the transfection for $24 \mathrm{~h}$. NC=negative control. ${ }^{*} \mathrm{P}<0.05$.
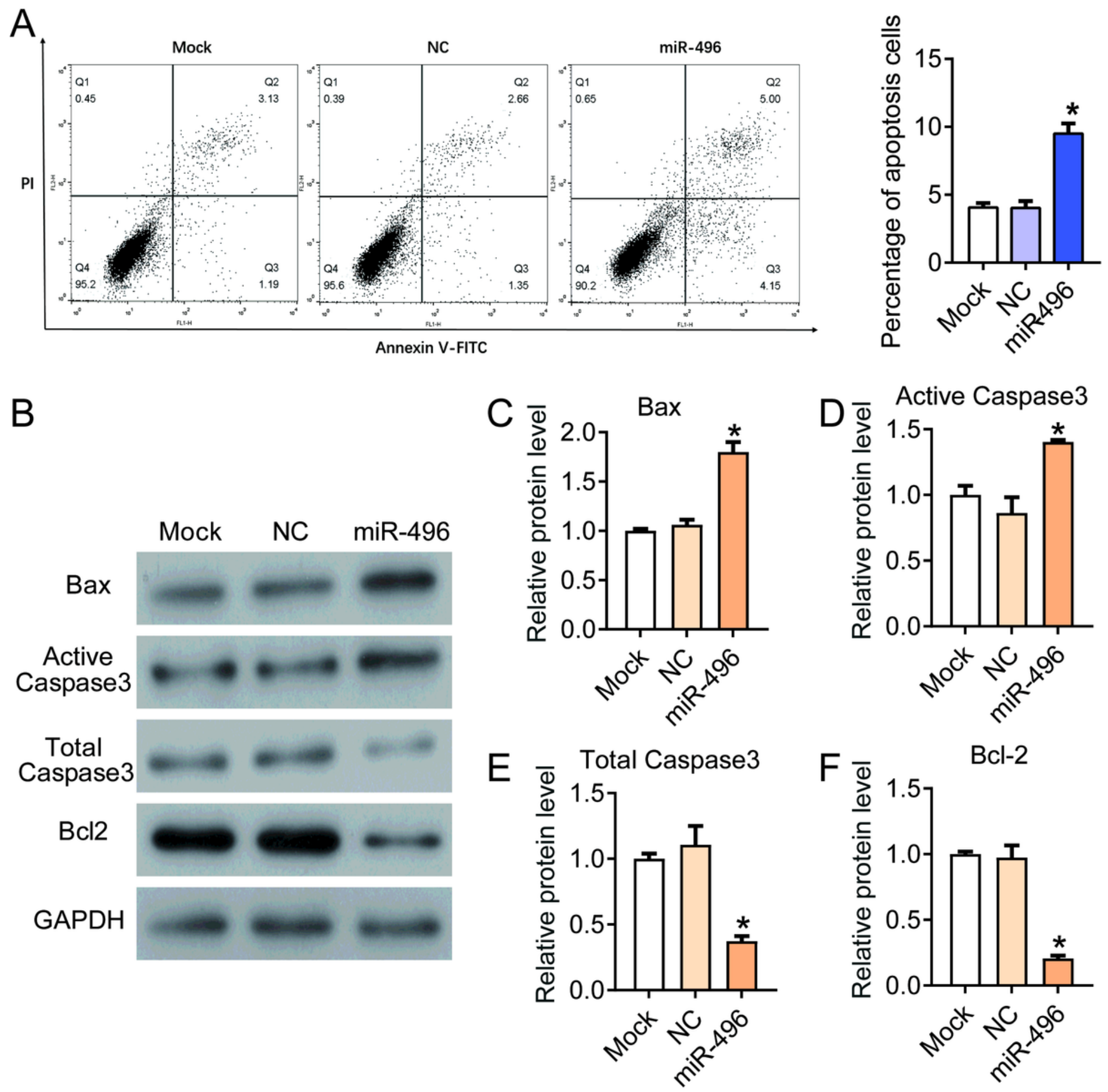

Figure 2

MiR-496 promoted the apoptosis in gastric cancer cells. A. Flow cytometry was performed to detect the apoptosis in AGS cells. B. The expression levels of apoptosis related protein Bax (C), Active Caspase3 (D), Total Caspase3 (E) and Bcl-2 (F) were detected by western blot. The relative protein levels were normalized to mock group. $\mathrm{NC}=$ negative control. ${ }^{*} \mathrm{P}<0.05$. 
A

Position 1250-1256 of LYN 3' UTR 5 ' . . UUUUUUGAUUUUAUUUU-UAAUACAC . . .

hsa-miR-496

II $\quad$ || 1111

CUCUAACCGGUACAUUAUGA
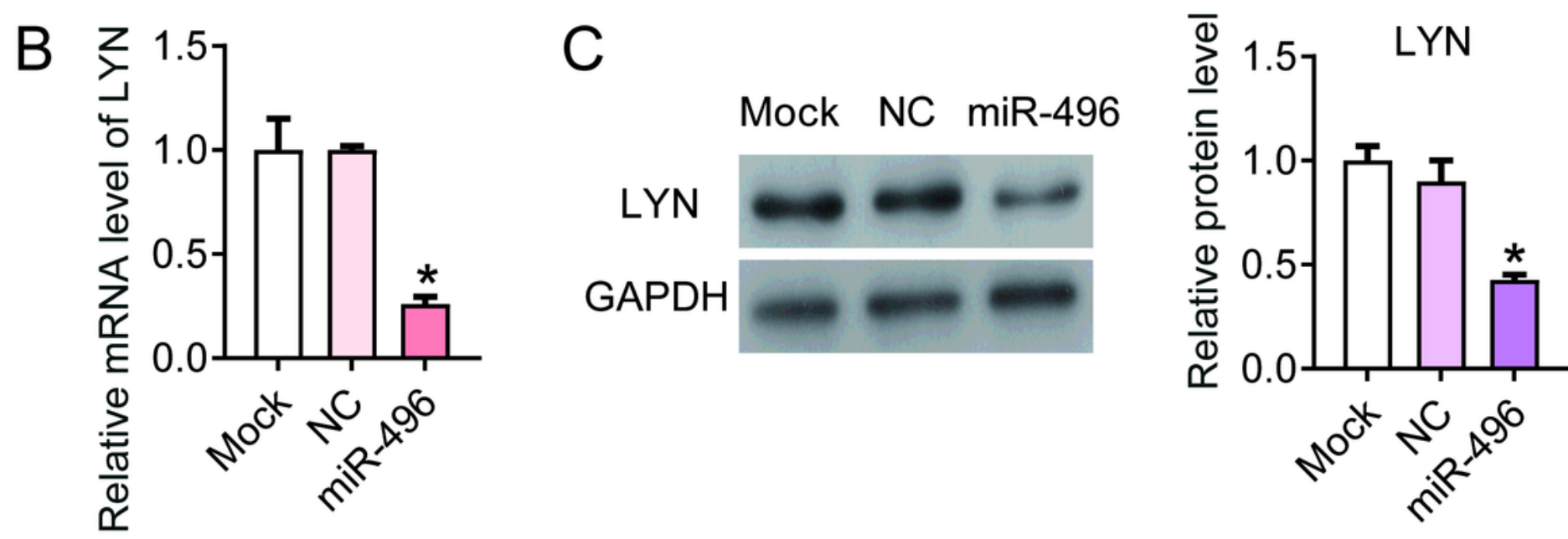

Figure 3

MiR-496 inhibited the expression of LYN in gastric cancer cells. A. According to the analysis on targetscan, there was a binding site between miR-496 and 3 'UTR of LYN. B. QPCR was performed to detect the level of LYN in AGS cells transfected with miR-496 mimics. Relative level of LYN was analyzed using 2- $\Delta \Delta \mathrm{Ct}$ method and normalized to mock group. $\mathrm{C}$. he expression levels of LYN was detected by western blot. The relative protein levels were normalized to mock group. $N C=$ negative control. ${ }^{*} P<0.05$. 
A

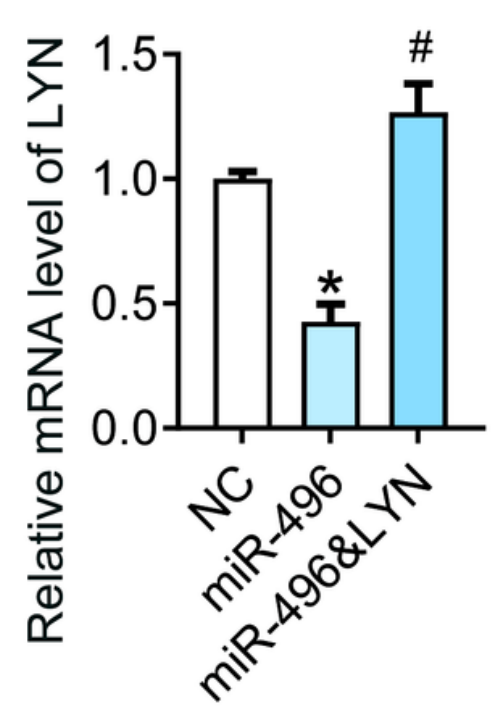

B The proliferation of AGS cells

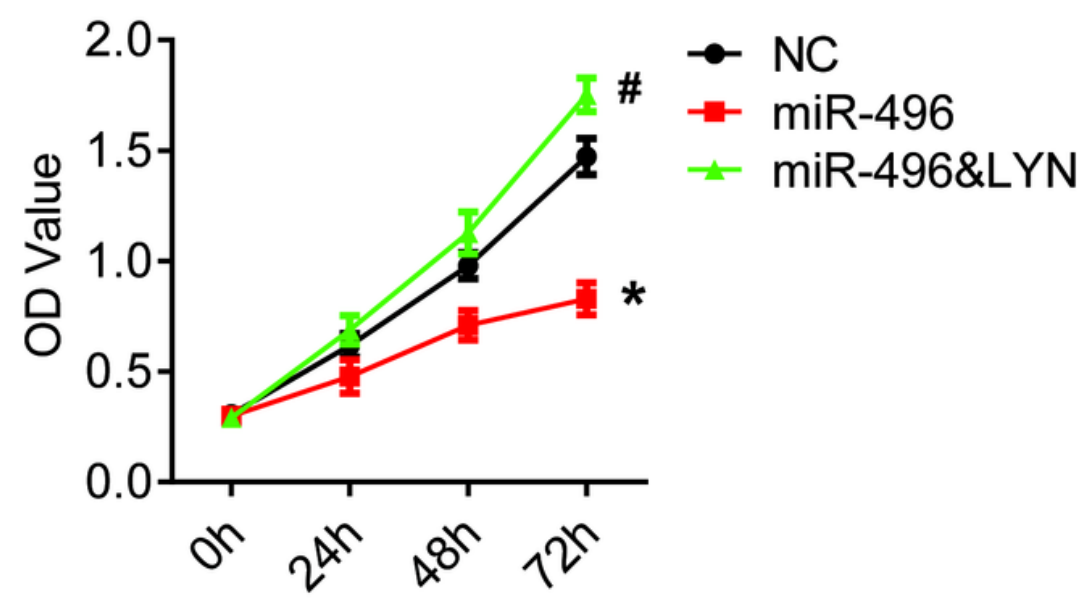

C
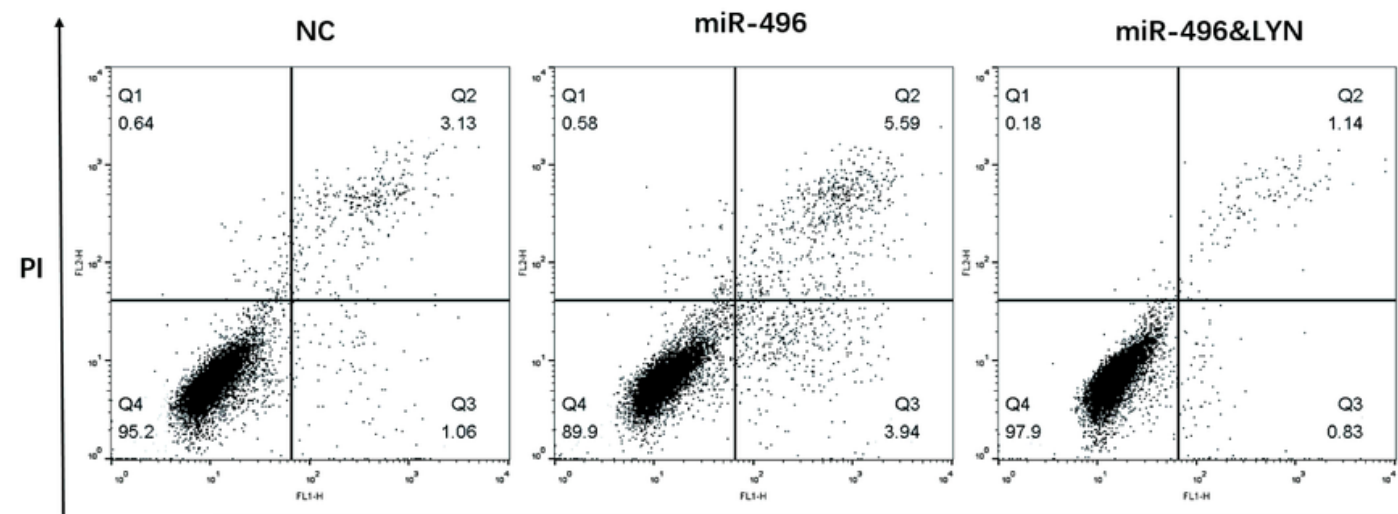

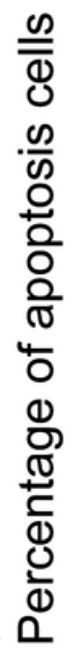

Annexin V-FITC

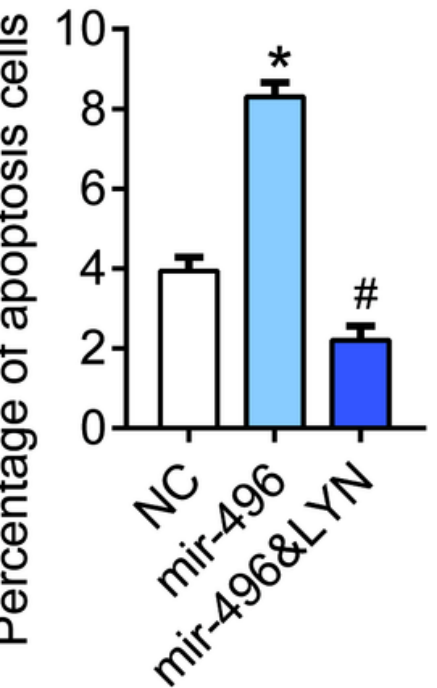

Figure 4

LYN blocked cell apoptosis induced by miR-496 in gastric cancer cells. A. QPCR was performed to detect the level of LYN in AGS cells transfected with miR-496 mimics or miR-496 mimics + LYN overexpression. B. the proliferation was detected by CCK8 assay. OD value $(450 \mathrm{~nm})$ was measured every $24 \mathrm{~h}$. C. Flow cytometry was performed to detect the apoptosis in AGS cells. NC=negative control. ${ }^{*} P<0.05 ; \# P<0.05$. 
A

NC miR-496 miR-496\&LYN

B

p-AKT

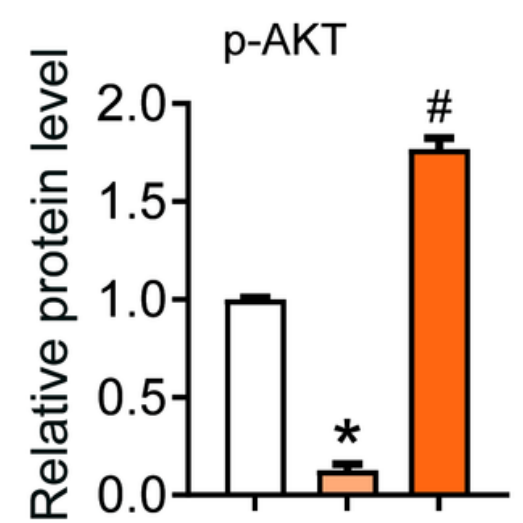

p-P70

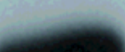

p-mTOR

Cyclin D1

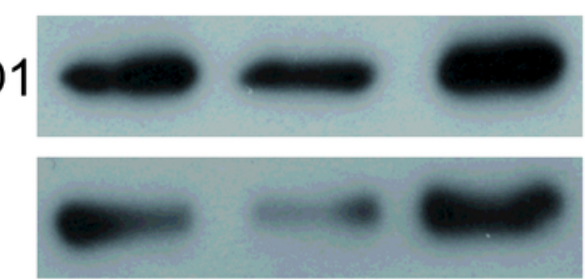

GAPDH
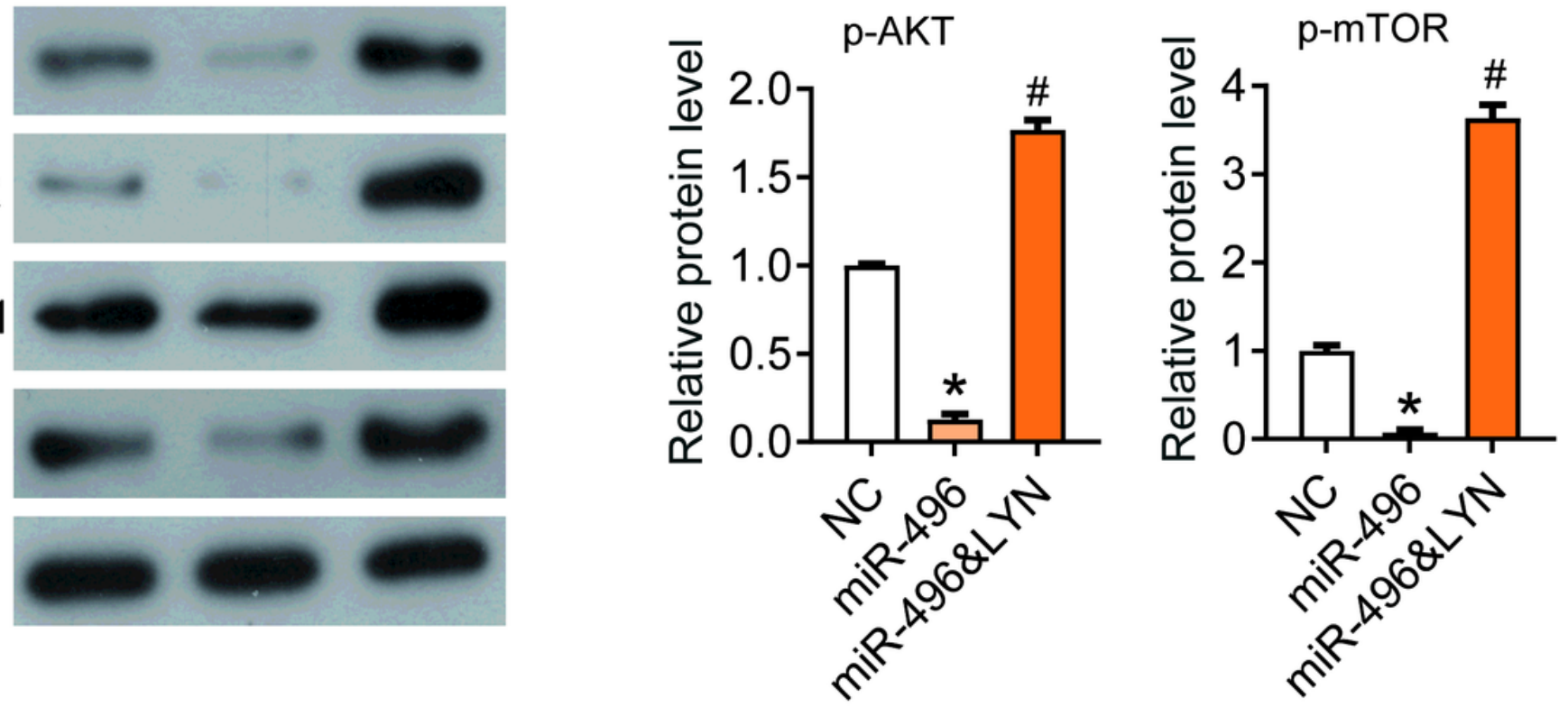

D
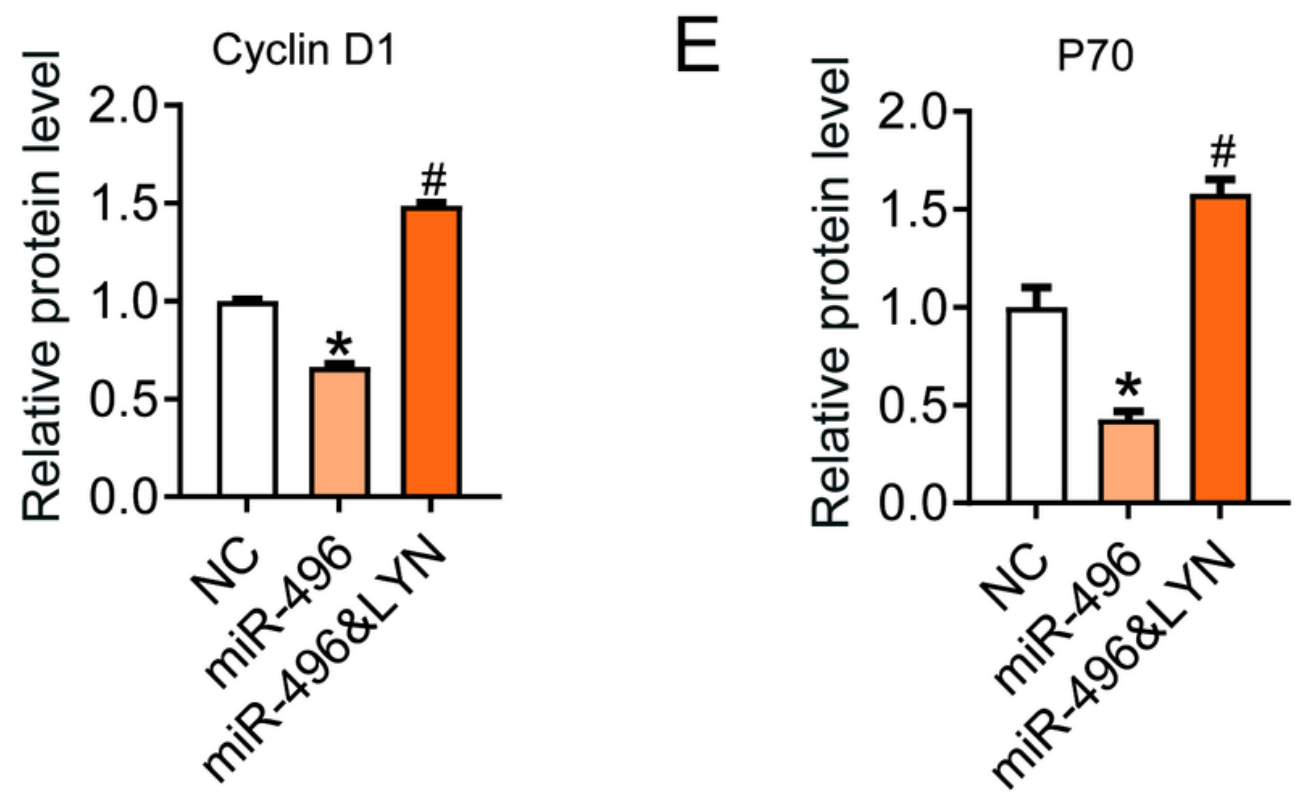

Figure 5

MiR-496 inhibited the AKT/mTOR signaling pathway by targeting LYN in gastric cancer cells. A. The expression levels of AKT/mTOR pathway related protein p-AKT (B), p-mTOR (C), Cyclin D1 (D), P70 (E) 'were detected by western blot. The relative protein levels were normalized to NC group. NC=negative control. * $\mathrm{P}<0.05 ; \# \mathrm{P}<0.05$. 